\title{
Incorporating cognitive retraining for management of individuals having cannabis dependence currently abstinent
}

\begin{abstract}
Cognitive deficits are one of the undesirable consequences of cannabis intake. A review of studies in this area was undertaken. It was found that individuals who consume cannabis suffer from widespread cognitive deficits. The level of deficits and degree of recovery are related to the chronicity, level, and age of onset of cannabis use. Some functions tend to be impaired for a long time and affect the process of recovery in individuals who try to remain abstinent. Neuroimaging studies in humans and studies in animals have shown that cannabis alters the brain structure and level of neurotransmitters. Cognitive deficits limit the capacity of individuals using cannabis to gain benefit from psychosocial management. Incorporating cognitive retraining with psychosocial management may facilitate better outcome.
\end{abstract}

Keywords: cannabis, cognitive functions, cognitive deficit, cognitive retraining, marijuana
Volume 3 Issue 5 - 2017

\author{
Amool R Singh, Masroor Jahan, Sarin \\ Dominic \\ Department of Clinical Psychology, Ranchi Institute of Neuro- \\ Psychiatry and Allied Sciences, India
}

\begin{abstract}
Correspondence: Amool R Singh, Professor, Department of Clinical Psychology, Ranchi Institute of Neuro-Psychiatry and Allied Sciences, Ranchi, Jharkhand, 834006, India, Email sisamool@yahoo.com
\end{abstract}

Received: February 27, 2017 | Published: July 03, 2017

\section{Introduction}

Cannabis is one among the most widely consumed drugs in the world. Studies indicate that almost $3 \%$ to $4 \%$ of the world's adult population abuses the drug (WHO, 1997). Higher doses over long term have been found to produce undesirable physical and psychological consequences, notably cognitive deficits. ${ }^{1}$ Subjective reports of deficits in memory, attention or concentration are common among cannabis users. ${ }^{2}$ A review of research findings on the nature and pattern of cognitive deficits reported in abstinent individuals, and factors determining the deficits. A comprehensive literature search was conducted on PubMed using the keywords cannabis, marijuana, cognitive deficits and retraining to identify relevant articles published between 1990 and 2016. A total of 21 empirical studies were obtained, mostly conducted in the United States and Europe.

\section{Neuro-cognitive functions in individuals with cannabis dependence who are currently abstinent}

Several neuropsychological studies conducted on individuals having cannabis abuse during consumption as well as at different periods of abstinence show alterations in cognitive functions. Researchers report that impairment occurs during the early period of abstinence i.e., up to 3 weeks. ${ }^{3}$ Cognitive functions is likely to improve over time, but the degree of recovery varies from individual to individual. Neuropsychological testing in individuals who use cannabis regularly shows impairment in memory, attention, ${ }^{4}$ abstraction, decision-making and executive functioning ${ }^{5,6}$ during initial stage of abstinence. Findings are inconsistent. Regarding reversibility of these deficits over the course of abstinence. Attention and concentration deficits resolves within a month of abstinence but subtle deficits in verbal memory, learning, mental speed and executive functions such as decision making, concept formation, set shifting and planning persist. ${ }^{1,7}$

\section{Determinants of level of cognitive deficits and recovery}

Mixed results on pattern of cognitive recovery in individuals who are abstinent from cannabis signal possibility that there are other variables (related and/or not related to cannabis use) moderating the recovery. Researchers have examined chronicity, dosage and age of onset in relation to recovery. Performance of Long term users was significantly poor on tests of cognitive functions during early abstinence. ${ }^{4}$ Number of lifetime marijuana use episodes was associated with greater cognitive deficits, suggesting a cumulative dose effect of marijuana use. ${ }^{6}$ Amount of use of cannabinoids was also found to be a significant factor determining level of deficits. ${ }^{1,8}$ A longitudinal study of cognitive functions from birth to adulthood, reported participants Hooper $^{8}$ with history of cannabis dependence prior to age of 18 showed neuropsychological decline over time. ${ }^{9}$ Early use of cannabis is likely to negatively influence neurodevelopmental process occurring in adolescence. ${ }^{10}$ Reduction in regional cerebral blood flow in PET scans ${ }^{11}$ and alteration in electrical activity as evidenced by fMRI studies ${ }^{12}$ particularly in the frontal lobe in adolescent cannabis users, indicate that there is a structural change.

\section{Neurobiological underpinnings of cognitive deficits in cannabis dependence}

Several studies examining neurophysiological changes and associated deficits in cognitive functions of individuals using cannabis have reported notable changes in brain's electrical activity, density of white and grey matter and brain metabolism in cannabis users while comparing to that of nonusers. ${ }^{13}$ Studies utilizing MRI techniques identified differences in regional brain volume, showed decreased volume in right Para-hippocampal gyrus and left parietal lobe, increased volume in pre-central gyrus and the right thalamus in cannabis users. ${ }^{14,15}$ Lower resting regional cerebral blood flow, decreased glucose uptake in certain regions of the brain, dysfunctional metabolic relationships between frontal and medial temporal regions ${ }^{16}$ were identified in individuals having chronic cannabis use. Reduction in regional cerebral blood flow in PET $\operatorname{scan}^{11}$ and alteration in electrical activity in $\mathrm{fMRI}^{12}$ were found in adolescent cannabis users. Developing neurons in the fronto-temporal regions were found to be affected in adolescents who initiate cannabis use at an earlier 
age. ${ }^{10}$ Cannabis was found to reduce the level of neurotransmitter acetylcholine in the prefrontal cortex. ${ }^{17}$ Since acetylcholine is essential for intact attention and memory functions, impairment of cognitive functions in individuals who use cannabis may be due to a decrease in the level of brain acetylcholine.

\section{Impact of cognitive deficits in psychological management of cannabis dependence}

Cognitive deficits, especially deficits in executive functions is likely to impair control of behaviour and keep individuals in the trap of addiction. Studies on patients who underwent psychological management for various substance dependence disorders have reported that poorer executive cognitive functions become a hurdle in the change process in patients, as patients with this difficulty are unaware of the severity of the problem and resort to denial. ${ }^{18,1}$ Patients with cannabis dependence who drop out from psychological management program were found to score lower than completers on measures of executive functions such as abstract reasoning and processing accuracy. ${ }^{2}$ Hence it can be inferred that, in order to prevent dropout of patients from therapy and to maximize the benefit of psychosocial management, cognitive deficits need to be remediated.

\section{Incorporating cognitive retraining strategies for better outcome}

Cognitive retraining is the process of retraining for improving cognitive functions through repeated practice, and is based on the principle of brain plasticity. ${ }^{20,21}$ Several methods and strategies have evolved in the past two to three decades, addressing various cognitive deficits experienced by patients with psychiatric and neurological problems. ${ }^{21}$ With regard to substance dependence, patients with alcohol disorders have shown improvement in cognitive functions as a result of retraining practices. ${ }^{22-25}$ Improvement of cognitive functions in those who underwent training showed increased psychological wellbeing and decrease in craving. ${ }^{26-31}$

\section{Conclusion}

Given that cognitive deficits is associated with cannabis use and the promising results of cognitive retraining in alcohol dependence, it is suggested that incorporating cognitive retraining alongside other psychosocial management and pharmacotherapy may be beneficial in the rehabilitation of cannabis users.

\section{Acknowledgements}

None.

\section{Conflict of interest}

The author declares no conflict of interest.

\section{References}

1. Crean RD, Tapert SF, Minassian A, et al. Effects of Chronic, Heavy Cannabis Use on Executive Functions. J Addict Med. 2011;5(1):9-15.

2. Aharonovich E, Brooks AC, Nunes EV, et al. Cognitive deficits in marijuana users: Effects on motivational enhancement therapy plus cognitive behavioral therapy treatment outcome. Drug Alcohol Depend. 2008;95(3):279-283.

3. Pope HG, Gruber AJ, Hudson JI, et al. Neuropsychological performance in long-term cannabis users. Arch Gen Psychiatry. 2001;58(10):909915.
4. Solowij N, Stephens RS, Roffman RA, et al. Cognitive functioning of long-term heavy cannabis users seeking treatment. JAMA. 2002;287(9):1123-1131.

5. Whitlow CT, Liguori A, Livengood LB, et al. Long-term heavy marijuana users make costly decisions on a gambling task. Drug Alcohol Depend. 2004;76(1):107-111.

6. Medina KL, Hanson K, Schweinsburg AD, et al. Neuropsychological functioning in adolescent marijuana users: Subtle deficits detectable after 30 days of abstinence. J Int Neuropsychol Soc. 2007;13(5):807-820.

7. Bolla KI, Brown K, Eldreth D, et al. Dose-related neurocognitive effects of marijuana use. Neurology. 2002;59(9):1337-1343.

8. Hooper SR, Woolley D, De Bellis MD. Intellectual, neurocognitive, and academic achievement in abstinent adolescents with cannabis use disorder. Psychopharmacology (Berl). 2014;231(8):1467-1477.

9. Meier MH, Caspi A, Ambler A, et al. Persistent cannabis users show neuropsychological decline from childhood to midlife. Proc Natl Acad Sci USA. 2012;109(40):2657E-2664E.

10. Ashtari M, Cervellione K, Cottone J, et al. Diffusion abnormalities in adolescents and young adults with a history of heavy cannabis use. $J$ Psychiatr Res. 2009;43(3):189-204.

11. O’Leary DS, Block RI, Koeppel JA, et al. Effects of smoking marijuana on brain perfusion and cognition. Neuropsychopharmacology. 2002;26(6):802-816.

12. Schweinsburg AD, Schweinsburg BC, Cheung EH, et al. FMRI response to spatial working memory in adolescents with comorbid marijuana and alcohol use disorders. Drug Alcohol Depend. 2005;79(2):201-210.

13. Smith A, Zunini R, Anderson R, et al. Impact of Marijuana on Response Inhibition: an fMRI Study in Young Adults. Journal of Behavioral and Brain Science. 2011;1(3):124-133.

14. Quickfall J, Crockford D. Brain neuro imaging in cannabis use: A review. J Neuropsychiatry Clin Neurosci. 2006;18(3):318-332.

15. Battistella G, Fornari E, Annoni JM, et al. Long-term effects of cannabis on brain structure. Neuropsychopharmacology. 2014;39(9):2041-2048.

16. Parkar SR, Ramanathan S, Nair N, et al. Cannabis dependence: Effects of cannabis consumption on inter-regional cerebral metabolic relationships in an Indian population. Indian Journal of Psychiatry. 2010;52(3):236-242.

17. Gessa GL, Casu MA, Carta G, et al. Cannabinoids decrease acetylcholine release in the medial-prefrontal cortex and hippocampus, reversal by SR 141716A. Eur J Pharmacol. 1998;355(2-3):119-124.

18. Blume AW, Marlatt GA. The role of executive cognitive functions in changing substance use: what we know and what we need to know. Ann Behav Med. 2009;37(2):117-125.

19. Le Berre AP, Pinon K, Vabret F, et al. Study of metamemory in patients with chronic alcoholism using a feeling-of-knowing episodic memory task. Alcohol Clin Exp Res. 2010;34(11):1888-1898.

20. Plaut DC. Relearning after damage in connectionist networks: Toward a theory of rehabilitation. Brain Lang. 1996;52(1):25-28.

21. Malhotra S, Bhatia MS, Rajender G, et al. Current Update on Cognitive Retraining in Neuropsychiatric Disorders. Delhi Psychiatry Journal. 2009;12(2):213-218.

22. Heinrichs RW, Levitt H, Arthurs A, et al. Learning and retention of a daily activity schedule in a patient with alcohol Korsakoff's syndrome. Neuropsychological Rehabilitation. 1992;2(1):43-58.

23. Goldstein G, Haas GL, Shemansky WJ, et al. Rehabilitation during alcohol detoxication in comorbid neuropsychiatric patients. J Rehabil Res Dev. 2005;42(2):225-234. 
24. Levine B, Schweizer TA, O'Connor C, et al. Rehabilitation of executive functioning in patients with frontal lobe brain damage with goal management training. Front Hum Neurosci. 2011;5:9 p.

25. Monteiro MFA, Bolognani SAP, Rivero TS, et al. Neuropsychological intervention in a case of Korsakoff's amnesia. Brain Impairment. 2011;12(3):231-238.

26. Rupp C, Kemmler G, Kurz M, et al. Cognitive remediation during treatment for alcohol dependence. J Stud Alcohol Drugs. 2012;73(4):625634.

27. Everitt BJ, Hutcheson DM, Ersche KD, et al. The orbital prefrontal cortex and drug addiction in laboratory animals and humans. Ann N Y Acad Sci. 2007;1121:576-597.
28. Fergusson D, Horwood JL. Early onset cannabis use and psychosocial development in young adults. Addiction. 1997;92(3):279-296.

29. Gruber AJ, Pope HG, Hudson JI, et al. Attributes of long-term heavy cannabis users: a case- control study. Psychol Med. 2003;33(8):14151422 .

30. Hart CL, van Gorp W, Haney M, et al. Effects of acute smoked marijuana on complex cognitive performance. Neuropsychopharmacology. 2001;25(5):757-765.

31. O'Hara C, Harrell M, Bellingrath E, et al. Cognitive Symptom Checklists. Psychological Assessment Resources, Inc, Odessa (FL), India; 1993. 